Mens

revue d'histoire intellectuelle de l'Amérique française

MENS

\title{
L'érection de la croix du mont Royal
}

\section{Gaston Côté}

Volume 7, numéro 1, automne 2006

URI : https://id.erudit.org/iderudit/1024222ar

DOI : https://doi.org/10.7202/1024222ar

Aller au sommaire du numéro

Éditeur(s)

Centre de recherche en civilisation canadienne-française

ISSN

1492-8647 (imprimé)

1927-9299 (numérique)

Découvrir la revue

Citer cet article

Côté, G. (2006). L'érection de la croix du mont Royal. Mens, 7(1), 47-72.

https://doi.org/10.7202/1024222ar

\section{Résumé de l'article}

L'érection de la croix sur le mont Royal en 1924 est riche de sens à la fois dans l'histoire de Montréal et celle du Québec. En entreprenant de l'ériger, la Société Saint-Jean-Baptiste de Montréal souhaite d'abord rappeler les gestes posés par Jacques Cartier et Paul Chomedey de Maisonneuve. Le monument participe aussi des célébrations de la fête de la Saint-Jean-Baptiste dont l'ampleur croît dans les années 1920, une période caractérisée par un regain du nationalisme. En outre, l'élévation du monument sur la montagne témoigne de l'avancée des francophones dans un secteur de Montréal traditionnellement associé à l'élite anglophone. Enfin, ce symbole important de la nationalité canadienne-française marque la volonté de prendre possession de cet espace symbolique et, plus globalement, de la ville tout entière. 


\title{
L'ÉRECTION DE LA CROIX DU MONT ROYAL ${ }^{1}$
}

\author{
Gaston Côté \\ Département d'histoire \\ Université de Montréal
}

\section{Résumé}

L'érection de la croix sur le mont Royal en 1924 est riche de sens à la fois dans l'histoire de Montréal et celle du Québec. En entreprenant de l'ériger, la Société Saint-Jean-Baptiste de Montréal souhaite d'abord rappeler les gestes posés par Jacques Cartier et Paul Chomedey de Maisonneuve. Le monument participe aussi des célébrations de la fête de la Saint-Jean-Baptiste dont l'ampleur croît dans les années 1920, une période caractérisée par un regain du nationalisme. En outre, l'élévation du monument sur la montagne témoigne de l'avancée des francophones dans un secteur de Montréal traditionnellement associé à l'élite anglophone. Enfin, ce symbole important de la nationalité canadienne-française marque la volonté de prendre possession de cet espace symbolique et, plus globalement, de la ville tout entière.

\section{Abstract}

By erecting a cross on Montreal's Mount Royal in 1924, the Société Saint-JeanBaptiste sought to emulate similar actions taken centuries before by Cartier and Maisonneuve. The erection of this profoundly symbolic monument was linked to Saint-Jean-Baptiste day celebrations and, more importantly, to a renewal of French Canadian nationalism. The erection of a cross on Mount Royal was viewed as a symbol of the advancement of French Canadians in an area of the city traditionally dominated by the English-speaking elite, and furthermore, as a symbol of the French Canadian possession of this symbolic space and of the city itself. 
Le 24 juin 1924, M ${ }^{\text {gr }}$ Emmanuel-Alphonse Deschamps, vicaire général du diocèse de Montréal, bénit la pierre angulaire de la croix que la Société Saint-Jean-Baptiste de Montréal (SSJBM) érige sur le sommet du mont Royal. Cette cérémonie clôt les festivités de la fête de la Saint-Jean-Baptiste. Amorcée en mai, la construction du monument se termine en septembre. La première illumination a lieu la veille de Noël de la même année.

Que souhaite alors commémorer la Société Saint-JeanBaptiste par ce geste ? D'une part, elle fait écho à la croix plantée par Jacques Cartier à Gaspé pour marquer la prise de possession du lieu au nom du roi de France, le 24 juillet 1534. Cartier est aussi lié à l'histoire de la montagne puisqu'en 1535, il l'aurait gravie et baptisée du nom de «mont Royal ». D'autre part, la croix commémore le projet de Paul Chomedey de Maisonneuve d'ériger une croix sur le mont Royal si la colonie naissante était épargnée par l'inondation de décembre 1642. Son souhait exaucé, Maisonneuve en éleva donc une sur la montagne le 6 janvier 1643 .

Depuis les années 1980, l'étude de la commémoration connaît un regain d'intérêt chez les historiens ${ }^{2}$. Les travaux produits depuis ont mis en lumière les différentes manières dont le passé a été utilisé pour légitimer un ordre social ou conforter le sentiment identitaire. Au tournant du $\mathrm{XX}^{\mathrm{e}}$ siècle, une vague de commémorations publiques déferle sur le monde occidental. Orchestrées par les dirigeants laïcs et religieux, les commémorations transmettent des messages ancrés dans le passé au moyen de somptueux défilés, de grands monuments, etc. Ces messages se présentent comme la réponse des élites sentant leur autorité menacée par l'industrialisation, la laïcisation, l'immigration, etc. ${ }^{3}$

Le Québec n'échappe pas à cette vague de commémoration. Alan Gordon a mis en lumière l'explosion d'actes com- 
mémoratifs qui se produit à Montréal de 1891 à 1930. Les élites anglophones et francophones se sont alors disputé l'espace public afin de façonner, par l'érection de marqueurs physiques dans la ville, la mémoire publique. Ces marqueurs constituent autant de témoins de leurs interprétations respectives du passé 4 .

À la même époque, la ville de Québec est le théâtre d'importantes commémorations célébrant le tricentenaire de Québec et les figures de Samuel de Champlain et de $\mathrm{M}^{\mathrm{gr}} \mathrm{de}$ Laval. Dans son étude de ces événements, Ronald Rudin soutient que la commémoration permet d'observer et de saisir l'évolution de la société québécoise. Il démontre que les fondateurs ont joué un rôle dominant dans la mémoire du Québec au tournant du $\mathrm{XX}^{\mathrm{e}}$ siècle parce qu'ils symbolisaient les racines d'une civilisation qui était parvenue à survivre malgré tous les obstacles dressés sur sa route ${ }^{5}$.

Parmi les monuments et les cérémonies commémoratives, la croix du mont Royal occupe un emplacement de choix qui lui confère une très grande visibilité, encore aujourd'hui. En dépit de sa prédominance dans le paysage montréalais, elle a très peu retenu l'attention des historiens. Dans son ouvrage sur l'histoire de la Société Saint-Jean-Baptiste de Montréal, Robert Rumilly aborde l'érection du monument. Selon lui, l'œuvre de la Société symbolise le lien intime existant entre l'histoire de Montréal et l'histoire de la Société SaintJean-Baptiste et entre la vie de Montréal et la vie de la Société Saint-Jean-Baptiste. ${ }^{6}$ Pour sa part, Alan Gordon estime que le geste commémoratif procédait bien davantage d'une volonté d'actualiser un événement inspirant de l'histoire de Montréal, en renouant avec l'esprit religieux des fondateurs de Montréal. L'auteur soutient aussi que la croix résulte des tentatives de la Société Saint-Jean-Baptiste de Montréal pour 
réclamer le mont Royal pour les résidants francophones de la ville. Malheureusement, Gordon développe très peu cette idée.

Paul-André Linteau, quant à lui, estime que l'érection de la croix du mont Royal et, plus spécialement, le défilé du 24 juin 1924 s'apparentent à des manifestations du réveil nationaliste des années 1920. Ce réveil nationaliste constitue alors une réaction de défense contre la menace que font peser sur la culture francophone à Montréal la domination économique des anglophones et l'envahissement culturel des Américains ${ }^{7}$.

La croix apparait donc riche de significations même si elle a peu retenu l'attention de chercheurs à ce jour. Dans un contexte de réaffirmation du nationalisme canadien-français, tel qu'il se manifeste dans les années 1920, elle se présente comme une étape importante du processus d'appropriation de la ville de Montréal par les francophones. Dans ce texte, nous souhaitons explorer cette dynamique en utilisant la couverture journalistique de l'événement. Comment l'érection du monument a-t-elle été interprétée dans la presse écrite ? A-telle été perçue comme partie intégrante du mouvement d'appropriation de la ville par les francophones? Pour tenter de répondre à ces questions, nous avons dépouillé La Patrie, Le Devoir, La Presse, Le Canada, The Montreal Daily Star et The Montreal Gazette en nous concentrant sur trois périodes clés de l'érection du monument. La première période dépouillée est le mois de mai 1924, alors qu'on annonce l'octroi d'un terrain par la Ville de Montréal. Elle correspond au début de la construction. La deuxième période est le mois de juin alors que l'on bénit la pierre angulaire de la croix lors de la fête de la Saint-Jean-Baptiste. Enfin, la dernière période est la semaine de Noël de la même année au cours de laquelle on procède à la première illumination du monument. Nous avons complété notre corpus d'articles en dépouillant la revue de la 
Société Saint-Jean-Baptiste de Montréal, La Revue nationale, afin d'en éclairer l'action et la vision.

L'entre-deux-guerres correspond à une période de regain du nationalisme canadien-français. Le nationalisme biculturel canadien à la Henri Bourassa du tournant du siècle fait alors place à un nationalisme proprement canadien-français, dont Lionel Groulx est la figure de proue ${ }^{8}$. Depuis le tournant du siècle, Henri Bourassa associe étroitement défense de la langue et défense la religion qu'il perçoit comme les deux faces d'une même médaille. Les menaces répétées à la langue française comme les crises scolaires ou l'affichage unilingue anglais viennent modifier les positions de ces leaders nationalistes. Au début des années 1920, Bourassa soutient qu'il faut d'abord défendre la religion. De son côté, Groulx, dont la ferveur catholique ne diminue aucunement, affirme davantage le caractère canadien-français de la nation. Sceptique face à l'avenir de la Confédération, Groulx devient le chef de file de ceux qui doutent et qui cherchent une alternative?.

Depuis les années 1860, les Canadiens français sont majoritaires à Montréa $1^{10}$. Si au cours des années 1920, ils améliorent leur niveau de vie, ils n'en demeurent pas moins une majorité « dominée ». Une proportion importante des francophones est sous-scolarisée et occupe des emplois peu qualifiés et peu rémunérateurs. L'économie de la ville est toujours contrôlée par la grande entreprise anglophone ce qui se reflète dans le visage anglais de la ville. Ce visage anglais est très prononcé au centre-ville où l'affichage est surtout unilingue anglais ${ }^{11}$.

Depuis la fin du XIX ${ }^{\mathrm{e}}$ siècle, de concert avec l'augmentation du poids démographique des francophones à Montréal, il se produit un mouvement d'appropriation territoriale de l'espace urbain par les francophones ${ }^{12}$. Comme l'épisode de l'érec- 
tion de la croix sur le mont Royal l'illustre, cette appropriation s'observe également dans l'espace symbolique qu'est la commémoration.

\section{Une croix sur la montagne : de l'idée à la construction}

L'idée d'ériger une croix sur le mont Royal n'est pas tout à fait nouvelle. C'est à l'occasion de la fête de la SaintJean-Baptiste de 1874 que l'abbé Alexandre-Marie Deschamps, curé de Saint-Sulpice, lance l'idée lors d'un sermon invitant à renouveler le geste de Maisonneuve, l'année même où le célèbre architecte du paysage de Central Park à New York, Frederick Law Olsmted, accepte le contrat d'aménagement d'un parc sur le mont Royal ${ }^{13}$. Toutefois, la proposition de Deschamps tombe dans l'oubli jusqu'à ce que la Société SaintJean-Baptiste de Montréal relance l'idée dans les années 1920.

Plusieurs sociétés ou associations participent aux entreprises de commémoration à Montréal durant la période faste des années 1891 à 1930. Les membres de ces sociétés choisissent alors les événements qui, à leurs yeux, méritent d'être commémorés. Parmi ces sociétés, la SSJBM occupe le premier rang du côté francophone, portée par son célèbre président Victor Morin, une des figures les plus actives dans la construction de la mémoire publique montréalaise ${ }^{14}$. Président de la SSJBM depuis 1915, le notaire Morin est le grand promoteur du projet. En 1923, un comité des finances est mis sur pied pour amasser les sommes nécessaires, mais l'entreprise s'avère ardue. La SSJBM est submergée par des demandes de toutes sortes comme le soutien financier d'écoles, d'hôpitaux, de paroisses francophones au Québec et au Canada. Le projet est donc reportét ${ }^{15}$.

Il refait surface en 1924, grâce à Morin qui le prend à sa charge. Il est d'emblée prévu que la croix, construite en béton armé, mesurera 100 pieds de hauteur et sera recouverte de 
granit blanc, de manière à refléter la lumière durant le jour. La nuit, elle sera illuminée par un système électrique ${ }^{16}$. Mais la campagne de financement piétine. Dans ce contexte difficile, Édouard-Zotique Massicotte, archiviste du district de Montréal et membre actif de la SSJBM, suggère sans succès à Morin d'abandonner le projet pour le remplacer par un autre jugé plus utile, tel le soutien à la construction d'hôpitaux ${ }^{17}$.

Pour financer le projet, Edmond Montet, un ami personnel de Morin, propose de lancer les élèves des écoles catholiques de Montréal dans une grande collecte. Les enfants vendraient des timbres à l'effigie de la croix et profiteraient ainsi de l'occasion pour acquérir de bonnes valeurs patriotiques $^{18}$. Les autórités scolaires acceptent. À la suite d'une rencontre entre les directeurs de la SSJBM et les membres du comité exécutif de la Ville de Montréal, cette dernière consent à céder un terrain de 100 pieds carrés, à même le parc du mont Royal. De plus, la Ville s'engage à faire les travaux de terrassement, à fournir le granit nécessaire et à aménager les alentours du site ${ }^{19}$.

La vente des timbres par les écoliers se termine en mars 1924 et permet d'amasser la somme de 10000 \$. Le conseil général de la SSJBM demande des plans pour la croix. Parmi les treize reçus, il opte pour celui du sulpicien Pierre Dupaigne. À la fête de la Saint-Jean-Baptiste, la construction n'en est encore qu'à ses débuts. La structure métallique est finalement terminée le 18 octobre, mais sans son recouvrement de granit. Elle aura coûté $11000 \$$ soit $1000 \$$ de plus que le produit de la collecte sans compter qu'il manque encore le système d'éclairage. À cette fin, Morin demande à la Montreal Light, Heat and Power de fournir gratuitement l'électricité, ce que la compagnie accepte de faire ${ }^{20}$.

Faute de moyens, cependant, la croix ne sera jamais recouverte de granit. Mais personne ne s'en formalise dans 
les journaux ni même au sein de la SSJBM. C'est que le projet, tout de même grandiose, est devenu réalité.

\section{La fête de la Saint-Jean-Baptiste de 1924}

La bénédiction de la pierre angulaire de la croix le 24 juin 1924 s'inscrit dans le cadre des célébrations de la fête de la Saint-Jean-Baptiste. Depuis la fin du XIX ${ }^{e}$ siècle, les organisateurs de tels actes commémoratifs font appel à des traditions relatives aux célébrations publiques canadiennes-françaises. Une façon d'utiliser l'espace public à ces occasions consiste à faire défiler les gens dans les rues en ayant recours aux formes particulières de processions reliées à la Fête-Dieu et à la Saint-Jean-Baptiste, fêtes dont les origines remontent au XVII ${ }^{e}$ siècle au Québec. Comme les Canadiens français avaient l'habitude d'occuper la rue durant ces fêtes, les organisateurs ont eu recours à ce « langage de représentation " pour les aider à raconter les histoires qu'ils souhaitent communiquer $^{21}$. Il importe donc de s'attarder aux fêtes de la Saint-JeanBaptiste de 1924.

En plus de l'érection de la croix, la Société projette d'organiser un grand congrès et un défilé, les trois projets devant former un ensemble coordonné. Massicotte est chargé du défilé. On décide de mettre à profit l'archiviste pour donner au défilé un cachet historique rigoureux. En confiant le défilé à une seule personne, on souhaite que les chars soient coordonnés sur le plan historique et sur le plan du message pour éviter l'hétéroclisme des défilés traditionnels. Massicotte s'entoure du dessinateur Jean-Baptiste Lagacé et du notaire Elzéar Roy, expérimenté en mise en scène théâtrale ${ }^{22}$.

Au printemps, le trio fait approuver par le conseil général de la SSJBM le thème du défilé qui consiste à mettre en valeur " ce que l'Amérique doit à la race française ». Le défilé doit rappeler l'exploration, l'évangélisation et la civilisation 
d'une grande partie du continent par la « race » française. Au total, 24 chars allégoriques sont construits ${ }^{23}$.

Les thèmes abordés lors du défilé concernent essentiellement la période de la Nouvelle-France. Ils mettent en gros plan quatre figures types de la présence française en Amérique du Nord : celles de l'explorateur, du fondateur, du colonisateur et du missionnaire, qu'incarne une pléiade de héros ${ }^{24}$. Les explorateurs sont représentés par le Père Marquette, Louis Jolliet, Cavelier de la Salle et de la Vérendrye, salués pour avoir découvert et exploré des territoires couvrant tout le Canada et les États-Unis. La « race » française fut donc la première présente sur le continent et elle a ouvert la voie aux Britanniques. Les fondateurs sont Champlain, Maisonneuve, Iberville et Blainville. Ceux-ci ont fondé les deux grandes villes québécoises, mais aussi de nombreuses villes américaines. Un char représente les États américains "que la grande République américaine, reconnait devoir à la race française ${ }^{25}$ »; un autre, les villes fondées par des Français ou des Canadiens au Canada. Un autre encore traite des provinces canadiennes sillonnées et colonisées en premier lieu par la « race française ». D'autres chars font la part belle aux colonisateurs comme Champlain, Hébert, Cavalier de la Salle et aux missionnaires représentés par les Martyrs canadiens qui ont jeté les bases de la civilisation. À la présentation des héros de la NouvelleFrance, on associe des valeurs qui en font des exemples à suivre. Ces héros sont chrétiens, entièrement dévoués à leur mission et attachés à la sauvegarde de la nationalité canadienne-française ${ }^{26}$.

Outre l'exaltation de la «race » qui vise à stimuler la fierté des Canadiens français, le défilé s'adresse aussi à l'« Autre ", c'est-à-dire à l'anglophone et à l'étranger. Selon La Revue nationale, le défilé "apprendra à nos compatriotes quelle dette de reconnaissance nous devons à ceux dont les 
noms devraient être gravés dans tous les cœurs canadiens ; elle inspirera même à nos concitoyens d'origine étrangère un respect profond pour ceux qui ont renouvelé au Canada les "gesta Dei per Francos"27".

Les journaux soutiennent que l'effet sur les spectateurs est de taille. Pour Le Devoir, le défilé a illustré de « façon douloureuse la profonde ignorance » dont témoignent les Canadiens français de leur propre histoire ${ }^{28}$. On relate qu'un ouvrier se serait écrié "sous une forme rude et pittoresque » devant le char des États américains fondés par des Canadiens ou des Français : «Après tout, les Canadiens ne sont pas plus bêtes que les autres puis, on est chez nous partout ici ${ }^{29} »$. Voilà qui va dans le sens de l'« exaltation de la race ».

Le défilé quitte le parc Lafontaine, emprunte les rues Cherrier, Saint-Hubert, Mont-Royal, Saint-Denis, puis le boulevard Saint-Joseph pour finalement se rendre au parc JeanneMance $^{30}$. On signale la présence de nombreux étrangers sur tout le parcours de la procession, des " compatriotes" venus des villes pratiquement "canadiennes » de la Nouvelle-Angleterre $^{31}$. La foule est importante : les différents journaux s'entendent pour l'évaluer à $300000^{32}$. La procession arrive au parc Jeanne-Mance vers les cinq heures. La foule demeure au pied de la montagne. Au sommet, $\mathrm{M}^{\mathrm{gt}}$ Deschamps se tient au milieu de personnalités dont $\mathrm{M}^{\mathrm{gr}}$ Forbes, évêque de Joliette, le sénateur Laurent-Olivier David, fraîchement honoré par la SSJBM, de nombreux échevins et des consuls. Au bas de la montagne, le maire de Montréal, Charles Duquette, les membres du conseil général de la SSJBM et les membres des sections qui ont défilé dans la procession et la foule attendent en silence $^{33}$. Dans le silence, l'officiant prononce les prières rituelles d'usage et trace sur chacun des côtés de la pierre un signe de croix. Puis, le maittre de chapelle entame le Magnificat. La foule au bas de la montagne attend le signal convenu 
pour répondre au chant puisque ni la foule ni l'entourage de $\mathrm{M}^{\mathrm{gr}}$ Deschamps ne peuvent se voir. Pour une raison ou une autre, la foule ne reçoit pas le signal. Finalement, un chœur d'enfants autour de l'officiant entame l'« Ô Canada ! » qui clôt la cérémonie ${ }^{34}$.

Enfin, la SSJBM organise un grand congrès national des Canadiens français au Monument national. En trois séances, le congrès doit réunir des francophones du Canada et des États-Unis. Afin de stimuler la lutte au Québec, Morin souhaite y entendre les communautés francophones hors Québec raconter leur combat. Vivant en contexte minoritaire dans les autres provinces canadiennes ou aux États-Unis, ces communautés démontrent pour Morin une grande capacité de résistance que les Canadiens français du Québec ne peuvent qu'admirer. Les Acadiens qui profitent des progrès réalisés depuis la fin du XIX ${ }^{\mathrm{e}}$ siècle sont offerts en exemple : "leurs rameaux dispersés sont venus se rattacher aux racines du sol ancestral et $[\ldots]$ ils ont grandi au point de diriger aujourd'hui les destinées de la province qu'ils habitent ${ }^{35} »$. Les Canadiens français de l'Ontario et de l'Ouest, de leur côté, tiennent bon alors qu'ils luttent « contre la persécution officielle » des gouvernements ${ }^{36}$. Enfin, les Franco-Américains représentent un cas particulièrement exemplaire, car non seulement ils préservent la langue française, mais aussi les valeurs associées à la nationalité canadienne-française. Aux yeux de Morin, les Américains ont pour caractère distinctif «le culte du veau d'or ${ }^{37} »$.

La première séance animée par Lionel Groulx porte sur «les devoirs actuels à l'égard de la race ». Sous la direction de Jean-Chrysostome Martineau, vice-président de l'ACJC, la deuxième séance porte sur la « résistance aux infiltrations étrangères ». Enfin, la dernière traite du " développement des relations entre les diverses branches de la famille canadienne- 
française » et est animée par Eugène Jalbert de Woonsocket, conseiller juridique de l'Union Saint-Jean-Baptiste d'Amérique et président de la Fédération catholique franco-américaine $^{38}$.

\section{Regards sur la croix}

Les articles dont nous disposons proviennent principalement du Devoir, quotidien dont les sympathies nationalistes sont bien connues. Durant ses premières années, c'est un journal de combat et un instrument de promotion des Canadiens français $^{39}$. Il faut aussi préciser que les articles de journaux ne nous livrent pas la réception populaire de l'événement, mais davantage une perception proche des élites nationalistes. La presse écrite n'en constitue pas moins un témoin privilégié de cette dynamique.

Toutes les réactions à la construction de la croix sont favorables. La couverture médiatique insiste particulièrement sur la démesure du projet. On rappelle sans arrêt ses 100 pieds de hauteur, ses six pieds d'épaisseur, sa base de 30 pieds carrés et son envergure de 81 pieds. À la fin de la construction, Le Devoir, avec beaucoup de détails, contribue à l'impression de démesure : «Environ deux mille pièces d'acier et dix mille boulons au poids total de vingt-sept tonnes, entrent dans la construction de la charpente d'acier [...]. Les seize piliers de base ont nécessité le forage dans le granit de trente-deux trous $^{40}$ ». Dans Le Canada, on fait valoir que la croix sera le point le plus élevé de la montagne; aucun arbre ne pourra en cacher la vue ${ }^{41}$. L'illumination, au soir du 23 décembre 1924, est considérée par Le Devoir comme un spectacle sans pareil dans les annales des trois siècles d'existence de la métropole ${ }^{42}$. La croix du mont Royal est un objet de fierté et d'orgueil pour le journal. 
Pour La Patrie, la croix rappelle "à ceux qui seraient tentés de l'oublier que la ville de Montréal fut fondée par des disciples de la croix, tout comme l'île d'Hochelaga, avait été découverte par un chrétien fervent ${ }^{43} »$. Le journal explique la nécessité de ce rappel au moment où le "cosmopolitisme " menacerait d'enlever à Montréal son caractère chrétien. Ceux qui seraient tentés de l'oublier sont à la fois les Canadiens anglais et les étrangers, ces derniers " qui se sont fondus dans la population montréalaise ${ }^{44} »$.

La croix est le symbole universel du christianisme. Cependant, les journaux font grand cas de la valeur symbolique de la croix spécifiquement pour les Canadiens français. Le Devoir, notamment, souligne l'importance de la tradition canadienne-française de planter des croix ${ }^{45}$. Au début des années 1920 , on recense quelque 200 croix uniquement sur l'île de Montréal. Celles-ci se sont multipliées durant la crise de la conscription, dont le souvenir est encore frais, afin de protéger les jeunes Canadiens français des recruteurs militaires. Dans un tel contexte, la croix prend valeur de symbole de résistance nationale ${ }^{46}$. Ainsi, le monument du mont Royal témoigne de la survivance et de la ténacité du peuple canadienfrançais :

Elle atteste la survivance étonnante du peuple canadien, né sous l'égide de la croix, protégé, développé par elle, grandissant en dépit des obstacles, et puisant dans l'âpreté même des luttes, comme la religion du Christ à travers les persécutions, la valeur nécessaire à la conservation de sa foi, de sa langue et de ses traditions ${ }^{47}$.

Dans la foulée des célébrations de la fête de la SaintJean-Baptiste de 1924, des nationalistes lancent un signal d'alarme. Ainsi, Omer Héroux se livre à cette réflexion: 
Nous ne vivons point dans une tour d'ivoire : cela veut dire que tous les vents mauvais qui soufflent sur le monde peuvent nous atteindre [...]. Il y a des Canadiens français $[. .$.$] qui [\ldots]$ passent à travers la vie sans avoir la moindre idée des difficultés que rencontre leur race, des dangers qui la menacent... ${ }^{48}$

Les dangers qui menacent la nation sont l'anglicisation, l'immigration, l'américanisation et l'apathie des Canadiens français. C'est à ce dernier élément que s'attaque la commémoration étudiée ici, car selon les élites nationalistes c'est l'apathie qui permet aux éléments étrangers d'altérer l'intégrité de la nationalité. La commémoration apparaît donc comme un outil pour éduquer les esprits. Il s'agit d'abord de faire comprendre aux Canadiens français que l'avenir de la nation est menacé et qu'il faut passer à l'action. Puis, il importe de démontrer à l'« Autre » son mécontentement et son refus d'abdiquer.

La peur de la perte de la langue et de l'intégrité ethnique inquiète. Une enquête de l'Association catholique de la jeunesse canadienne (ACJC) qui a démontré selon le vice-président de la SSJBM, Guy Vanier, "jusqu'à quel point lamentable nous subissons de toute part des influences néfastes pour la pureté de notre langue maternelle et l'intégrité de notre particularisme ethnique ${ }^{49} »$. Face à ces inquiétudes teintées de xénophobie, les nationalistes justifient l'importance du peuple canadien-français par sa valeur qu'il tient de sa culture, de sa mission religieuse, de son passé glorieux, etc. ${ }^{50} \mathrm{Plu}$ sieurs journaux reprennent la phrase émise par la SSJBM, qui résume bien le message général du défilé : «L'Amérique septentrionale a été colonisée par trois races européennes. Entre celles-ci, la race canadienne-française réclame une large part de souvenirs, à cause de l'influence chrétienne et civilisatrice qu'elle a exercée sur une immense étendue du continent ${ }^{51}$. » 
Le peuple canadien-français a pris le relais de la civilisation française en Amérique et on lui attribue le rôle de peuple missionnaire de la pensée française et catholique ${ }^{52}$.

Des solutions sont proposées comme l'éducation du public pour assurer la poursuite de la mission des Canadiens français, et l'action des nationalistes est principalement dirigée vers la sensibilisation des Canadiens français ${ }^{53}$. C'est ainsi que Morin justifie la tenue des célébrations de la Saint-JeanBaptiste :

$\mathrm{Ah}$ ! Pourquoi ? C'est qu'il faut donner un symbole aux aspirations de notre race; c'est qu'il faut rappeler de temps à autre aux ouvriers de l'œuvre nationale qu'ils ont une mission à remplir, qu'ils doivent travailler jour par jour et sans relâche en vue du but à atteindre, qu'ils doivent suppléer au nombre par l'activité, par la constance de l'effort s'ils veulent former un peuple qui soit digne de prendre place au conseil des nations ${ }^{54}$ !

Les élites nationalistes considèrent que c'est par une éducation patriotique que la nation pourra surmonter les épreuves auxquelles elle fait face. Et c'est dans le passé que les Canadiens français trouveront l'inspiration nécessaire. Il s'agit donc de démontrer que la nation canadienne-française doit obtenir en Amérique du Nord la place que lui confère son rôle historique dans le développement du continent, son rôle civilisateur dans une contrée sauvage et impie. C'est ce passé qui fait des Canadiens français un peuple fondateur et il leur incombe de s'en montrer dignes. Présenté par le biais du défilé, ce passé est si glorieux que les Canadiens français ne peuvent demeurer dans un état d'apathie.

Le défilé représente aussi une occasion pour valoriser la nation canadienne-française au sein du Canada et des ÉtatsUnis. Cela traduit, d'une part, les difficultés que les francophones vivent au Canada et, d'autre part, celles qu'ils éprou- 
vent aux États-Unis. Cela illustre aussi que la nation canadienne-française des années 1920 ne connait pas les frontières provinciales, bien sûr, pas plus que la frontière canadoaméricaine $^{55}$. Pour les élites nationalistes, le défilé prend une importance toute particulière :

[II] sera une saisissante protestation de toute la race française contre le flot des calomnies étrangères ; protestation plus éloquente que tous les articles de revues ou de journaux, la seule qui n'admettra aucune réplique. Elle nous vengera des mépris et de bien des injures. Ce sera enfin une sorte de réhabilitation nationale dans la gloire du passés ${ }^{56}$.

Une autre façon de lutter contre l'apathie des Canadiens français est de faire de la participation aux manifestations nationalistes un devoir ${ }^{57}$. La fête de la Saint-Jean-Baptiste n'est pas encore officielle en 1924, mais un mouvement en ce sens est particulièrement fort cette année-là. Ainsi, on rappelle que la « race » anglaise a institué de nombreuses fêtes publiques officielles et qu'il faut, en contrepartie, reconnaitre l'existence de la "race » canadienne-française en Amérique. On recherche l'équivalent pour les Canadiens français et cela passe par la mobilisation : «il faut d'abord que nous démontrions aux autorités que le projet de transformer le 24 juin en fête officielle est bien le vœu unanime de la population canadienne-française $e^{58} \%$. Il en va de même pour la participation au défilé et à la cérémonie de bénédiction. La participation est ouverte à tous, riches ou pauvres, jeunes ou vieux, et doit être une expression de patriotisme et de foi ${ }^{59}$. Et il s'agit d'un devoir :

C'est notre grand jour de fête et il faut que tous ceux qui ont du sang canadien-français dans les veines aient assez de patriotisme pour y prendre part, ne fut-ce [sic] au moins que par leur présence. On nous a reproché trop souvent de mépriser et d'oublier notre passé. Ce 
serait une honte que de rester dans l'apathie dans une pareille circonstance. [...]. L'étranger y verra la preuve de notre volonté de vivre ${ }^{60}$.

À l'époque, la participation à la fête de la Saint-JeanBaptiste prend de plus en plus d'importance. Les fêtes sont teintées de ce vigoureux élan et les résultats commencent à se faire sentir. Le 24 juin 1924 tombe un mardi et certains commerces acceptent de fermer leurs portes. Mais on craint que d'autres commerces ne profitent de la fermeture de leurs concurrents. Le Devoir et La Patrie demandent alors aux industriels, commerçants et hommes d'affaires de fermer boutique, au moins pour l'après-midi, afin de donner la chance à leurs employés de remplir "un véritable devoir patriotique ${ }^{61}$ ». Le 23 juin, La Patrie publie le nom des maisons qui ont accepté de participer au mouvement afin de les féliciter et de souligner leur sacrifice, tout en promettant d'en faire autant pour les entreprises qui leur emboîteront le pas $^{62}$. Le journal demande aussi aux Canadiens français de s'abstenir de tout achat afin de ne pas pénaliser les commerçants qui respecteront la consigne. Certains commerces font de la publicité annonçant qu'ils seront fermés pour la fête de la Saint-Jean-Baptiste ${ }^{63}$. La maison Birks, de son côté, n'annonce pas de fermeture pour le 24 juin, mais souligne l'importance de la fête et s'y associe en reconnaissant que le français est la langue maternelle de la majorité de son personnel ${ }^{64}$.

\section{La croix et l'espace urbain}

Le monument, la cérémonie de bénédiction et le défilé ont comme caractéristique d'être très visibles dans la ville. En ce sens, le monument et les célébrations nous apparaissent ș'inscrire dans le vaste mouvement d'appropriation du territoire montréalais par les francophones, en cours depuis la fin du $\mathrm{XIX}^{\mathrm{e}}$ siècle. Depuis cette époque, la présence des fran- 
cophones dans l'espace public et symbolique ne cesse de s'étendre vers des secteurs traditionnellement réservés aux élites anglo-protestantes, comme le mont Royal ${ }^{65}$.

Depuis la deuxième moitié du XIX ${ }^{\mathrm{e}}$ siècle, les parcs, en tant qu'espaces destinés à l'ensemble des populations urbaines, deviennent des lieux publics de plus en plus importants ${ }^{66}$. Depuis sa création par Frederick Law Olmsted, le parc du mont Royal est doté d'un caractère quasi sacré qui découle, d'une part, des caractéristiques physiques et naturelles du site, de ses liens avec l'histoire de Montréal et, d'autre part, du travail d'exclusion que les élites effectuent pour s'approprier le lieu au détriment d'autres groupes. Dans l'aménagement et l'utilisation du mont Royal, les élites francophones, en particulier les élus locaux, font concurrence aux élites anglo-protestantes pour s'approprier ce symbole de pouvoir que représente la montagne: "Dominer la montagne, c'est aussi une manière de dominer la ville à distance ${ }^{67} \%$. De même, ériger un symbole représentant la nationalité canadienne-française au sommet de la montagne, c'est affirmer la prise de possession du lieu et de la ville.

C'est Victor Morin qui choisit l'emplacement de la croix avec l'aide d'un ingénieur de la Ville de Montréal ${ }^{68}$. Son choix est révélateur des intentions des promoteurs de la croix. Ainsi, l'emplacement ne constitue pas tout à fait le point le plus élevé de la montagne. Du point de vue est de la ville, cependant, elle parait effectivement sise au sommet. Cet emplacement fait en sorte que le monument domine la ville du côté où il est visible pour le plus grand nombre. De plus, la croix ne s'intègre pas au parc où elle est érigée. Son environnement immédiat, qui n'a pas été aménagé, ne permet pas de la mettre en valeur : elle est un élément insolite dans le parc ${ }^{69}$.

Pourtant, le plan retenu de l'abbé Pierre Dupaigne prévoyait des plates-formes d'observation situées dans les bras 
de la croix et un bâtiment recouvrant la base de la structure métallique et permettant d'y monter ${ }^{70}$. Ce projet aurait permis d'harmoniser le monument avec son contexte, celui d'un parc urbain fort populaire. Mais la réalisation complète du plan n'était pas essentielle. La caractéristique première de la croix est d'abord et avant tout sa grande visibilité. C'est toute la montagne et même l'île de Montréal qui lui sert de cadre. Les occasions sont nombreuses où, avec fierté, la couverture médiatique insiste sur le fait que la croix est visible de jour comme de nuit, et ce, de très loin :

Du sommet l'œil embrasse un panorama idéal, avec la vieille ville immense rayonnant à perte de vue, la plaine fertile ornée du ruban d'argent que le grand fleuve lance depuis les lacs bleus jusqu'à la mer ; ici les tons violets des montagnes isolées de l'est et là-bas la chaîne des Laurentides que dorent les rayons du soleil couchant ${ }^{71}$.

L'emplacement confère donc au monument un sens particulier. De la même manière que l'on plante un drapeau sur une place forte, l'érection d'une grande croix conçue comme un symbole de résistance nationale des Canadiens français au sommet du mont Royal représente la conquête d'un espace physique et symbolique qui permet d'affirmer que Montréal est avant tout une ville catholique et française : "Montréal, deuxième ville française et première ville catholique $^{72} »$. Ce n'est donc pas uniquement le parc du mont Royal qui est revendiqué pour les francophones, mais la ville toute entière.

En plus, la croix est à cheval sur trois espaces; la ville, la nature et le ciel. Il est alors facile de lui conférer une personnalité mystique. De cet endroit, la croix surplombe la ville et s'en détache dans un type d'espace sacré qui lie le peuple au divin : « ...élevant ses bras d'amour sur le peuple qui s'agite à ses pieds : puissante, majestueuse et sereine... ${ }^{73} \%$. 
L'utilisation de l'espace urbain lors de la cérémonie de bénédiction de la croix utilise la morphologie du paysage. Nombreuse, la foule qui a suivi le défilé, accompagnée de différents corps (zouaves, vétérans de la Grande Guerre, etc.) demeure silencieuse au pied de la montagne. Elle est à l'écart du rituel de bénédiction qui se déroule au sommet dans la clairière aménagée pour la croix. Là-haut, se trouve l'évêque qui effectue des rites religieux. Autour de lui, des élites politiques locales, des élites nationalistes et des consuls assistent en silence. Les deux groupes qui ne peuvent se voir sont unis par le chant sacré émanant du boisé et descendant vers la foule (rappelons ici que contrairement à ce qui était prévu, la foule n'a pu répondre).

Par ailleurs, se développe au même moment, entre les fidèles et la montagne, un lien strictement religieux, cette fois, un lien entièrement dépouillé d'aspects nationalistes et profanes. Depuis 1904, le populaire frère André et quelques ouvriers ont construit un petit oratoire en l'honneur de saint Joseph sur l'un des versants du mont Royal. En 1924, les autorités ecclésiastiques entreprennent la construction d'une énorme basilique sur le site. La notoriété du frère André est à son apogée ${ }^{74}$. Le site attire déjà de nombreux pèlerins venus des paroisses de Montréal ${ }^{75}$.

Enfin, mentionnons le début des travaux de construction du nouveau campus de l'Université de Montréal au flanc du mont Royal en 1928 pour remplacer ses installations de la rue Saint-Denis ${ }^{76}$. La montagne perd encore plus, durant la période, de ses liens avec l'élite anglo-protestante pour se retrouver davantage dans l'espace francophone.

\section{$* * *$}

Au-delà du sens donné par ses promoteurs, la croix du mont Royal se révèle être particulièrement riche de significa- 
tions. C'est bien le sommet de la montagne que la SSJBM s'approprie au nom des francophones. Qui plus est, la croix s'inscrit dans la poursuite d'un mouvement déjà bien enclenché dans ce secteur de la ville. Tant dans la presse francophone que dans la presse anglophone (bien que cette dernière s'y attarde très peu), les réactions sont favorables à l'érection du monument ${ }^{77}$. À l'époque, le projet n'a donc pas suscité de polémique. Serait-ce parce qu'il y a désormais acceptation du fait francophone dans ce secteur de la ville, contrairement à ce qui prévalait à la fin du XIX ${ }^{\mathrm{e}}$ siècle ? Il est permis de le croire. L'érection de la croix apparait donc comme une étape dans le processus d'identification avec le lieu, une tendance qui se confirme avec une autre célébration commémorative d'importance : les fêtes du tricentenaire de Montréal de 1942 qui ont lieu dans le parc du mont Royal ${ }^{78}$. En ce sens, l'érection de la croix sur le mont Royal peut être considérée comme un jalon significatif dans le mouvement d'appropriation de la ville par les francophones.

En outre, l'étude du monument permet de percevoir le nationalisme des années 1920 dans son inscription montréalaise, qui se manifeste à travers l'importance que prend la fête de la Saint-Jean-Baptiste. La croix du mont Royal et les célébrations entourant sa bénédiction représentent ainsi un épisode, à l'échelle montréalaise, du processus menant à l'institutionnalisation de la fête de la Saint-Jean-Baptiste. Comme nous l'avons vu, la fête devient jour férié l'année suivante.

Contrairement à d'autres monuments érigés à la même époque, la croix du mont Royal n'a pas cessé, depuis, d'être chargée de sens, sans doute en raison de la grande visibilité que lui confèrent sa taille et son emplacement. Aujourd'hui, elle demeure une puissante icône qui identifie instantanément 
Montréal. En somme, la croix est le témoin immobile d'un sens, pour sa part, sans cesse mouvant.

\section{NOTES}

${ }^{1} \mathrm{La}$ rédaction de ce texte n'aurait pas été possible sans le soutien financier du CRSH. Je tiens également à remercier madame Michèle Dagenais qui m’a grandement aidé par ses commentaires judicieux et ses corrections ainsi que les évaluateurs anonymes de Mens.

2 Voir en particulier Pierre Nora, dir., Les lieux de mémoire, Paris, Gallimard, 1984, 3 vol. et Eric Hobsbawm et Terence Ranger, dir., The Invention of Tradition, Cambridge, Cambridge University Press, 1983, 322 p.

${ }^{3}$ Ronald Rudin, L'bistoire dans les rues de Québec. La célébration de Champlain et de $M^{\text {rr }}$ de Laval 1878-1908, Sainte-Foy, Les Presses de l'Université Laval, 2005, pp. 2-3. On ne peut passer sous silence l'ouvrage d'Henry Vivian Nelles, L'bistoire spectacle. Le cas du tricentenaire de Québec, Montréal, Boréal, 2003, 428 p. Pour Nelles, la commémoration est un art qui repose sur une duperie intentionnelle. Il affirme que le tricentenaire de Québec a tissé l'illusion d'une nation fondée sur un passé plausible (p. 372).

${ }^{4}$ Alan Gordon, Making Public Pasts: The Contested Terrain of Montreal's Public Memories, 1891-1930, Montréal, McGill-Queen's University Press, 2001, 233 p.

${ }^{5}$ Rudin, L'bistoire dans les rues de Québec.

${ }^{6}$ Robert Rumilly, Histoire de la Société Saint-Jean-Baptiste de Montréal, Montréal, L'Aurore, 1975, p. 316.

${ }^{7}$ Paul-André Linteau, Histoire de Montréal depuis la Confédération, deuxième édition, Montréal, Boréal, 2000, p. 321.

${ }^{8}$ Susan Mann Trofimenkoff, Action française: French Canadian Nationalism in The Twenties, Toronto, University of Toronto Press, 1975, pp. 3-6.

'Yvan Lamonde, Histoire sociale des idées au Québec, 1896-1929, vol. 2, Montréal, Fides, 2004, pp. 272-273. Sur le nationalisme des années 1920 voir Frédéric Boily, Lapensée nationaliste de Lionel Groulx, Sillery, Septentrion, 2003, 234 p. ; Michel Bock, Quand la nation débordait les frontières. Les minorités françaises dans la pensée de Lionel Groulx, Montréal, Éditions Hurtubise HMH, 2004, $452 \mathrm{p}$.

${ }^{10}$ Linteau, Histoire de Montréal depuis la Confédération, p. 123. 
${ }^{11}$ Ibid., pp. 319-320.

${ }^{12}$ Michèle Dagenais, "Saisir les dynamiques d'un espace urbain : le parc LaFontaine, ses usages et ses publics ", dans Serge Jaumain et Paul-André Linteau, dir., Vivre en ville. Montréal et Bruxelles aux XIX ${ }^{e}$ et $X X^{c}$ siècles, Bruxelles, P.I.E. Peter Lang, 2006.

${ }^{13}$ David Bellman, « Frederick Law Olmsted et un plan pour l'aménagement du Mont-Royal / Frederick Law Olmsted and a Plan for Mount Royal Park ", David Bellman, dir., Mont-Royal: Montréal / Mount Royal: Montreal, Montréal, Musée McCord, 1977, p. 31.

${ }^{14}$ Gordon, Making Public Pasts, pp. 49-71.

${ }^{15}$ Rumilly, Histoire de la Société Saint-Jean-Baptiste de Montréal, pp. 307-308.

${ }^{16}$ Victor Morin, « La croix du Mont-Royal », La Revue nationale, janvier 1924, p. 6.

${ }^{17}$ Rumilly, Histoire de la Société Saint-Jean-Baptiste de Montréal, p. 310.

${ }^{18}$ Ibid.

${ }^{19}$ Anonyme, «La croix du Mont-royal », Le Devoir, 5 mai 1924, p. 4.

${ }^{20}$ Rumilly, Histoire de la Société Saint-Jean-Baptiste de Montréal, pp. 307-316.

${ }^{21}$ Rudin, L'bistoire dans les rues de Québec, pp. 5-9, 45.

${ }^{22}$ En 1926, Édouard-Zotique Massicotte et Elzéar Roy publient un livresouvenir sur les processions de 1924 et 1925 présentant chacun des chars : Processions de la Saint-Jean-Baptiste, Montréal, Librairie Beauchemin, 1926, 313 p.

${ }^{23}$ Rumilly, Histoire de la Société Saint-Jean-Baptiste de Montréal, pp. 309-313.

${ }^{24} \mathrm{Au}$ sujet du héros dans la commémoration, voir l'article de Fernande Roy, "Une mise en scène de l'histoire. La fondation de Montréal à travers les siècles ", Revue d'histoire de l'A mérique française, vol 46, n 2 (été 1992), pp. 7-36.

${ }^{25}$ Anonyme, «La procession de 1924 », La Revue nationale, juin 1924, p. 173.

${ }^{26}$ Ibid.

${ }^{27}$ Anonyme, "Les enseignements de la fête nationale », La Revue nationale, juin 1924, pp. 182-183.

${ }^{28}$ Anonyme, "La grande procession allégorique d'hier après-midi », Le Devoir, 25 juin 1924, p. 2. 
${ }^{29}$ Ibid.

${ }^{30}$ Anonyme, « Programme général des fêtes 1924 », La Revue nationale, juin 1924, p. 174.

${ }^{31}$ Anonyme, « M ${ }^{\mathrm{gr}} \mathrm{A}$.-E. Deschamps bénit la pierre angulaire de la croix du Mont-Royal », La Patrie, 25 juin 1924, p. 11.

${ }^{32}$ Gustave Comte, "Lendemain de fête nationale », La Patrie, 25 juin 1924, p. 18.

${ }^{33}$ Robert Rumilly, Histoire de la Société Saint-Jean-Baptiste de Montréal, p. 313.

${ }^{34}$ Anonyme, « $\mathrm{M}^{\mathrm{gr}} \mathrm{A}$.-E. Deschamps bénit la pierre angulaire de la croix du Mont-Royal », La Patrie, 25 juin, p. 11.

${ }^{35}$ Victor Morin, "Les enseignements de la fête nationale », La Revue nationale,

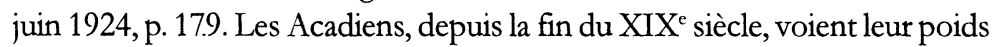
démographique s'accroître et participent de plus en plus à la vie politique du Nouveau-Brunswick. Cela s'accompagne de la montée du sentiment identitaire acadien contemporain. Jacques Paul Couturier, Un passé composé. Le Canada de 1850 à nos jours, Moncton, Éditions d'Acadie, 2000, pp. 33, 111-112, 209.

${ }^{36}$ Morin fait ici référence aux conflits scolaires en Ontario et dans l'Ouest. Voir Couturier, Un passé composé, pp. 99-104.

${ }^{37}$ Victor Morin, "Les enseignements de la fête nationale », La Revue nationale, juin 1924, p. 179.

${ }^{38}$ C'est Elphège Daignault qui devait présider cette séance, mais pour une raison ou une autre, il dut s'absenter. Rumilly, Histoire de la Société Saint-JeanBaptiste de Montréal, p. 315.

${ }^{39}$ Beaulieu, André et Jean Hamelin, La presse québécoise des origines à nos jours (1896-1910), Sainte-Foy, Presses de l'Université Laval, 1973, p. 330.

4) Anonyme, " La croix du Mont-Royal est presque terminée », Le Devoir, 16 septembre 1924, p. 1.

${ }^{41}$ Anonyme, « La ville accorde un terrain pour la croix du Mont-Royal », Le Canada, 5 mai 1924, p. 8.

${ }^{42}$ Anonyme, "La messe de minuit », Le Devoir, 26 décembre 1924, p. 2.

${ }^{43}$ Anonyme, " Pourquoi une croix sur le sommet du Mont-Royal », La Patrie, 9 juin 1924, p. 8.

${ }^{44}$ Ibid. 
${ }^{45}$ Anonyme, «La croix de M. de Maisonneuve », Le Devoir, 24 mai 1924, p. 1. ${ }^{46}$ Gordon, Making Public Pasts, pp. 99-101.

${ }^{47}$ Anonyme, [sans titre], La Presse, 24 décembre 1924, p. 3.

${ }^{48}$ Omer Héroux, « Pour la St-Jean-Baptiste », Le Devoir, 23 juin 1924, p. 2.

${ }^{49}$ Guy Vanier, « Nos séances d'étude », La Revue nationale, juin 1924, p. 193.

${ }^{50}$ Mann Trofimenkoff, Action française, p. 114.

${ }^{51}$ Anonyme, «La procession de 1924 », La Revue nationale, juin 1924, p. 167.

${ }^{52}$ Philippe Demers, "L'amour de la patrie », La Revue nationale, juin 1924, p. 187.

${ }^{53}$ Mann Trofimenkoff, Action française, p. 115.

${ }^{54}$ Victor Morin, " Les enseignement de la fête nationale », La Revue nationale, juin 1924, p. 178.

${ }^{55}$ À propos des minorités françaises hors Québec voir Bock, Quand la nation débordait les frontières.

${ }^{56}$ A. Gagnon, "Chronique de la société », La Revue nationale, mai 1924, p. 156.

${ }^{57}$ Selon Anatole Vanier, secrétaire général de L'Action franşaise, l'idée d'officialiser la Saint-Jean-Baptiste est lancée lors du premier congrès de la langue française au Canada en 1912. En 1916, la SSJBM se lance dans la promotion et décide d'entreprendre des démarches auprès du gouvernement provincial. En mai 1924, le Conseil municipal de Montréal et l'Union des municipalités adoptent des résolutions en ce sens. La fête est finalement officialisée en février 1925. Anatole Vanier, " Notre fête nationale ", L'Action française, mai 1925, pp. 301-302.

${ }^{58}$ Anonyme, "La Saint-Jean-Baptiste doit être fête nationale », La Patrie, 13 juin 1924, p. 9.

${ }^{59}$ Anonyme, « Pourquoi une croix sur le sommet du Mont-Royal », La Patrie, 9 juin 1924, p. 8.

${ }^{60}$ Anonyme, "Les fêtes de la St-Jean Baptiste se continuent ", Le Devoir, 24 juin 1924, p. 8.

${ }^{61}$ Anonyme, "La Saint-Jean-Baptiste doit être fête nationale », La Patrie, 13 juin 1924 , p. 9. 
${ }^{62}$ Anonyme, «Les maisons canadiennes-françaises qui fermeront leurs portes demain ", La Patrie, 23 juin 1924, p. 1.

${ }^{63}$ Publicité de Dupuis Frères, Le Devoir, 23 juin 1924, p. 8.

${ }^{64}$ Publicité d'Henry Birks \& Sons Limited, Le Devoir, 23 juin 1924, p. 8.

${ }^{65}$ Voir Gordon, Making Public Pasts, et Dagenais, « Saisir les dynamiques d’un espace urbain ».

${ }^{66}$ Michèle Dagenais. «Entre tradition et modernité : espaces et temps de loisirs à Montréal et Toronto au XX ${ }^{\mathrm{c}}$ siècle ", Canadian Historical Review, vol. 82, n² (juin 2001), pp. 311-312.

${ }^{67}$ Ibid.

${ }^{68}$ Rumilly, Histoire de la Société Saint-Jean-Baptiste de Montréal, p. 316.

${ }^{69}$ En 1998, Le Groupe d'intervention urbaine déplorait le manque d'aménagement au pied de la croix : « compte tenu de sa grande valeur symbolique et culturelle pour les Montréalais, on est surpris de la retrouver clôturée à la base. On n'y retrouve ni information expliquant son histoire, ni même un simple banc pour se reposer" (Groupe d'intervention urbaine de Montréal, La montagne en question, Montréal, Ville de Montréal, 1998, p. 16).

7) Ibid., p. 22.

${ }^{71}$ Anonyme, [sans titre], La Presse, 24 décembre 1924, p. 3.

${ }^{72}$ Ibid.

${ }^{73}$ Ibid.

${ }^{74}$ Tom Faulklner, "André, Frère », L'encyclopédie canadienne, http//:www.thecanadianencyclopedia.com, consulté le 12 novembre 2005.

${ }^{75}$ Henri-Paul Bergeron, Le Frère André c.s.c. L'apôtre de Saint-Joseph, Montréal, Oratoire Saint-Joseph du Mont-Royal, 1938, pp. 78-79.

${ }^{76}$ Paul-André Linteau, René Durocher, Jean-Claude Robert et François Ricard, Histoire du Québec contemporain, tome 2, Le Québec depuis 1930, Nouvelle édition révisée, Montréal, Boréal, 1989, p. 104.

77 Voir, par exemple, Anonyme, "Cross on Mountain », The Montreal Daily Star, 24 décembre 1924, p. 21.

${ }^{78}$ Voir Harold Bérubé, « Commémorer la ville : une analyse comparative des célébrations du centenaire de Toronto et du tricentenaire de Montréal », Revue d'histoire de l'Amérique française, vol. 57, $\mathrm{n}^{\circ} 2$ (automne 2003), pp. 209-236. 